\title{
Diferencias en la frecuencia de experiencias emocionales positivas y negativas en mujeres con distintos tipos de trastornos de la conducta alimentaria
}

\author{
Aitziber Pascual-Jimeno*, Pello Apodaca-Urquijo, Itziar Etxebarria-Bilbao y Maria Soledad Cruz-Sáez
}

Universidad del País Vasco

\begin{abstract}
Resumen: Con el objetivo de conocer la experiencia emocional de las mujeres con trastornos de la conducta alimentaria (TCA), analizamos la frecuencia con la que mujeres con distintos tipos de TCA tienen diversas experiencias emocionales positivas y negativas. Participaron 98 mujeres con TCA y 100 mujeres controles. De las mujeres con TCA, 17 tenían bulimia nerviosa $(\mathrm{BN})$ purgativa, otras $17 \mathrm{BN}$ no purgativa, 42 cumplían la mayoría de los criterios del DSM-IV-TR para una BN y 22 cumplían la mayoría de los criterios para una anorexia nerviosa (AN). Se diseñó ad hoc la Escala de Frecuencia de Experiencias Emocionales Positivas y Negativas. Los resultados mostraron que las mujeres que experimentaban emociones positivas menos frecuentemente y emociones negativas más frecuentemente eran las que cumplían la mayoría de los criterios para una AN. Por otra parte, las diferencias emocionales más claras se daban entre los casos que cumplían la mayoría de los criterios para una $\mathrm{AN}$ y los que cumplían la mayoría de los criterios para una BN. Estos resultados, además de tener implicaciones clínicas importantes, sugieren la necesidad de seguir estudiando este tema para confirmar si, como apuntan estos resultados, es necesario revisar la categoría de trastornos no especificados del DSM-IV-TR.

Palabras clave: Trastornos de la conducta alimentaria; emociones; experiencias emocionales; frecuencia.
\end{abstract}

\section{Introducción}

Desde que Bruch (1973) y Way (1993) documentaran la experiencia de emociones como la culpa, el miedo a la desaprobación y sentimientos de ineficacia en sus pacientes con anorexia nerviosa (AN), han sido muchos los autores que se han interesado en analizar la relación existente entre las emociones y los trastornos de la conducta alimentaria (TCA) (Arnow, Kenardy y Agras, 1992; Overton, Selway, Strongman y Houston; 2005; Schmidt y Treasure, 2006). De hecho, numerosos autores definen el propio trastorno alimentario como un intento desadaptativo de regular o escaparse de estados afectivos que son negativos para la propia persona (Alpers y Tuschen-Caffier, 2001; Arnow et al., 1992; Corstorphine, 2006; Heatherton y Baumeister, 1991; Moreno, 2003; Rodríguez, Mata y Moreno, 2007; Stice, Nemeroff y Shaw, 1996; Waters, Hill y Waller, 2001). En este sentido, la restricción alimentaria cumpliría la función de reducir las emociones negativas a través de un mecanismo de aprendizaje por refuerzo negativo, de forma que la restricción proporcionaría una respuesta de escape de dichas emociones negativas (Alpers y Tuschen-Caffier, 2001; Moreno, 2003; Waters et al., 2001). Por otra parte, el atracón se utilizaría como medio de distracción o alivio de los estados de ánimo negativos (Rodríguez et al., 2007).

Entre los autores que han tratado de establecer el papel concreto que desempeñan las emociones en los TCA, po-

* Dirección para correspondencia [Correspondence address]: Aitziber Pascual. Universidad del País Vasco. Facultad de Psicología. Avenida Tolosa, no 70. 20018. San Sebastián, Guipúzcoa (España). E-mail: aitziber.pascual@ehu.es
Title: Differences in the frequency of positive and negative emotional experiences in women with different types of eating disorders

Abstract: With the aim of exploring the emotional experience of women suffering from eating disorders (EDs), we analyzed the frequency with which women with different types of EDs have diverse positive and negative emotional experiences. Participants were 98 women with EDs and 100 controls. Of the women with EDs, 17 had purgative bulimia nervosa (BN), 17 had non-purgative BN, 42 fulfilled the majority of DSM-IV-TR criteria for $\mathrm{BN}$ and 22 fulfilled the majority of criteria for anorexia nervosa (AN). The Positive and Negative Emotional Experience Frequency Scale was designed ad boc for the study. The results revealed that women who experienced positive emotions less frequently and negative emotions more frequently were those who fulfilled the majority of criteria for AN. Also, the clearest emotional differences were observed between those who fulfilled the majority of criteria for $\mathrm{AN}$ and those who fulfilled the majority of criteria for $\mathrm{BN}$. In addition to having important clinical implications, these results also suggest the need to continue studying this question in order to confirm whether (as the results suggest) it is necessary to review the DSM-IV-TR non-specific disorder category.

Key words: Eating disorders; emotions; emotional experiences; frequency.

demos distinguir dos posiciones principales. Según una primera posición, las emociones actuarían como antecedentes o causas de los TCA (p. ej., Pascual et al., 2011a); y, según una segunda posición, éstas serían una consecuencia de los propios TCA (p.ej., Pells, 2005). Asimismo, hay autores que defienden ambas posiciones. Según estos autores, las emociones desempeñan un importante papel tanto en el desarrollo como en el mantenimiento de los TCA (p. ej., Schmidt y Treasure, 2006).

Uno de los problemas con los que nos encontramos a la hora de establecer el papel que desempeñan las emociones en los TCA es que en la mayoría de los estudios se han utilizado muestras no clínicas (Overton et al., 2005). En este tipo de estudios se ha encontrado una relación entre las emociones y las conductas de TCA (principalmente conductas bulímicas). A la hora de interpretar esta relación, de nuevo, nos encontramos con las mismas posiciones mencionadas anteriormente: mientras algunos autores hablan de las emociones como antecedentes o "causas" (p. ej., Arnow et al, 1992), otros hablan de "consecuencias" (Burney e Irwin, 2000). Por ejemplo, Arnow y sus colaboradores (1992) encontraron que las emociones más fuertemente asociadas a las conductas bulímicas eran la ansiedad, la depresión y la ira. Por otra parte, Burney e Irwin (2000) encontraron que los problemas alimentarios no estaban relacionados con la tendencia a la vergüenza y a la culpa en sentido global, sino tanto con la vergüenza y la culpa en contextos alimentarios como con la vergüenza asociada al cuerpo. En este sentido, concluyeron que la vergüenza y la culpa eran una consecuencia de los problemas alimentarios.

La mayoría de los autores que han estudiado la experiencia de emociones en los TCA ha analizado muy pocas emo- 
ciones. A continuación, se mencionan algunos de los resultados más interesantes hallados en este sentido. Por ejemplo, se ha encontrado que las personas con TCA, en comparación con las personas sin TCA, experimentan más depresión y ansiedad (Breaux y Moreno, 1994), más impulsividad (Claes, Vandereycken y Vertommen, 2002; Fahy y Eisler, 1993; Nagata, Kawarada, Kiriike e Iketani, 2000) y más hostilidad (Tiller, Schmidt, Ali y Treasure, 1995). Asimismo, también se ha encontrado que las personas con TCA, en comparación con las personas sin estos trastornos, tienen menos autoestima (Viñuales, Fernández, Jiménez, Turón y Vallejo 2001) y menor fuerza de voluntad (Shapiro, Blinder, Hagman y Pituck, 1993).

Uno de los pocos estudios que ha analizado la experiencia de un conjunto de emociones en una muestra clínica de TCA es el de Overton y sus colaboradores (2005). Estos autores utilizaron una muestra de 55 personas con TCA (AN y BN) y 100 personas controles. Para analizar la experiencia emocional utilizaron la Differential Emotions Scale (DES) (Izard, 1972) que evalúa la frecuencia con la que se experimentan 10 emociones básicas: dos positivas (interés y alegría), una neutra (sorpresa) y 7 negativas (miedo, rabia, asco, angustia, desprecio, vergüenza y culpa). Los resultados del estudio revelaron que, en comparación con las personas del grupo control, las personas con TCA experimentaban significativamente con mayor frecuencia sorpresa, asco, desprecio, vergüenza y culpa. Los autores concluyeron, en la misma línea de lo mencionado anteriormente, que las personas con TCA utilizan las conductas de TCA para manejar sus estados emocionales y que está dinámica podría constituir un importante factor de mantenimiento de los propios trastornos.

El estudio arriba señalado analizaba la experiencia emocional de personas con TCA en general, sin distinguir entre diferentes tipos de TCA. Sin embargo, estudiar las diferencias existentes en la experiencia emocional de los distintos TCA es importante, ya que dentro de esta categoría el DSMIV-TR (American Psychiatric Association, 2002) incluye trastornos muy diferentes entre sí (AN restrictiva, AN compulsiva/purgativa, BN purgativa, BN no purgativa y Trastorno de la conducta alimentaria no específicado, TCANE) y diversos estudios revelan que existen diferencias importantes en la experiencia emocional de las personas con distintos tipos de TCA. Por ejemplo, se ha encontrado que, en comparación con las personas con $\mathrm{AN}$, las personas con $\mathrm{BN}$ experimentan más depresión y ansiedad (Norman y Herzog, 1983) y más hostilidad (Forbush y Watson, 2006). Asimismo, en varios estudios se ha encontrado que las personas con BN muestran mayor impulsividad que las personas con AN (Claes et al., 2002; Fahy y Eisler, 1993; Nagata et al., 2000).

Por otra parte, a la hora de analizar la experiencia emocional de las personas con TCANE nos encontramos con un problema. Esta categoría, en la que se incluyen los trastornos alimentarios que no cumplen los criterios para un trastorno de la conducta alimentaria específico ( $\mathrm{AN}$ o $\mathrm{BN}$ ), ha recibido numerosas críticas. Según Williamson y sus colaborado- res (2002) es una especie de "cajón de sastre" en el que se incluyen muchos y muy diversos casos. Teniendo esto en cuenta, en un estudio reciente, Pascual y sus colaboradores (2011b) decidieron estudiar si había diferencias en diversas variables emocionales (como la alexitimia, la actitud negativa hacia la expresión emocional, la percepción negativa de las emociones, la regulación emocional, etc.) entre distintos tipos de TCA, analizando los casos que no cumplían todos los criterios para una AN y los casos que no cumplían todos los criterios para una $\mathrm{BN}$ (a partir de ahora, los casos de $\mathrm{AN}$ inespecífica y de $\mathrm{BN}$ inespecífica) de forma separada. Además de por estos trastornos inespecíficos, la muestra del citado estudio también estaba formada por personas con BN purgativa y con $\mathrm{BN}$ no purgativa. Los resultados mostraron que las diferencias emocionales más claras se daban entre los casos con $\mathrm{AN}$ inespecífica y los casos con $\mathrm{BN}$ inespecífica. En este sentido, los autores concluyeron que mezclar los trastornos inespecíficos hubiese supuesto perder información muy valiosa acerca de cada uno de estos trastornos.

Teniendo en cuenta todo lo señalado, en el presente estudio nos propusimos estudiar la experiencia emocional, concretamente, la frecuencia de experiencias emocionales positivas y negativas, de las personas con TCA, analizando los distintos tipos de TCA de forma separada. Estudiar esta cuestión nos parece importante no sólo para confirmar empíricamente lo que los profesionales clínicos ven a diario en su trabajo, sino también para tratar de entender algo más el papel que pueden estar desempeñando las emociones en estos trastornos y, consecuentemente, ayudar a mejorar, en la medida de lo posible, los programas de tratamiento que se aplican en estos trastornos.

Planteamos cuatro hipótesis concretas. Por una parte, supusimos que las mujeres con TCA experimentarían en general experiencias emocionales positivas con menor frecuencia y experiencias emocionales negativas con mayor frecuencia que las mujeres sin dichos trastornos. Asimismo, concretando un poco más la hipótesis anterior, planteamos que las mujeres con TCA, en comparación con las mujeres sin dichos trastornos, se sentirían más frecuentemente ansiosas, con sentimientos de inferioridad, con sentimientos de odio hacia sí mismas, culpables, enfadadas consigo mismas y decepcionadas consigo mismas. Por otra parte, planteamos que, entre las mujeres con los distintos tipos de TCA, serían las mujeres con $\mathrm{AN}$ las que experimentarían experiencias emocionales positivas con menor frecuencia y experiencias emocionales negativas con mayor frecuencia. Esta hipótesis no la teníamos muy clara, ya que en varios estudios (Forbush y Watson, 2006; Norman y Herzog, 1983) se han encontrado resultados contrarios a lo planteado en ella. Sin embargo, la observación clínica sí nos mostraba datos a favor de nuestra hipótesis y decidimos ponerla a prueba. Por último, teniendo en cuenta los resultados de otros estudios (Claes et al., 2002; Fahy y Eisler, 1993; Nagata et al., 200), planteamos que las mujeres con $\mathrm{BN}$ mostrarían mayor impulsividad que las mujeres con $\mathrm{AN}$. 


\section{Método}

\section{Participantes}

Participaron en el estudio 98 mujeres con TCA (Media de edad $=22.2, D T=5.87)$ y 100 mujeres controles (Media de edad $=21.34, D T=4.54)$ de Guipúzcoa, Vizcaya, Álava y Navarra. De las mujeres con TCA, 17 cumplían todos los criterios diagnósticos del DSM-IV-TR (American Psychiatric Association, 2002) para una BN purgativa y otras 17 todos los criterios para una BN no purgativa. Por otra parte, 22 cumplían la mayoría de los criterios del citado manual (concretamente, 3 de los 4 criterios establecidos) para una AN y 42, la mayoría de los criterios (concretamente, 4 de los 5 criterios establecidos) para una BN. Teniendo en cuenta los resultados obtenidos en el estudio de Pascual y sus colaboradores (2011b), señalados anteriormente, decidimos no mezclar en una única categoría los casos que no cumplen todos los criterios para un trastorno específico ( $\mathrm{AN}$ o $\mathrm{BN}$ ), como se hace en el DSM-IV-TR (American Psychiatric Association, 2002) con la categoría TCANE, sino incluir los casos que tengan mayor similitud con la $\mathrm{AN}$ en una categoría ("AN inespecífica") y los casos que tengan mayor similitud con la BN, en otra ("BN inespecífica").

El grupo control estaba formado por mujeres que no cumplían los criterios para el grupo de TCA, que no habían sido nunca diagnosticadas de un TCA, que no estaban recibiendo tratamiento psicológico, que tenían un índice de masa corporal $\leq 30$ (un índice $>30$ se considera sobrepeso crónico u obesidad grado II) y que habían obtenido una puntuación $\leq$ al percentil 85 en el Cuestionario de Salud General (Goldberg e Hillier, 1979).

La muestra para el grupo de TCA fue obtenida de diferentes centros específicos para los TCA (Asociación contra la Anorexia y la Bulimia de Euskadi, Servicio Vasco de Salud y Clínica Quirón de San Sebastián); y para el grupo control, de diversos institutos de educación secundaria del País Vasco y de Navarra y de varios centros universitarios de la Universidad del País Vasco y de la Universidad Pública de Navarra. La evaluación de estas muestras se realizó durante los años 2007 y 2008.

\section{Instrumentos}

En el cuestionario que rellenaron las participantes se solicitaban datos sociodemográficos como la edad y la ocupación. Posteriormente, para asignar la muestra clínica con TCA a los distintos grupos de TCA, se incluían en formato pregunta los criterios del DSM-IV-TR (American Psychiatric Association, 2002) para los diferentes TCA; asimismo, con el mismo objetivo, se requerían datos de peso y altura de la persona. Para establecer el grupo de control, además de las preguntas relativas al peso y la altura, se planteaban una serie de preguntas relativas a si habían sido alguna vez diagnosticadas de un TCA y a si estaban recibiendo tratamiento psicológico por alguna causa. Además, para establecer este gru- po, se incluía el Cuestionario de Salud General (Goldberg e Hillier, 1979). Finalmente, para evaluar la frecuencia con la que las participantes experimentaban diversas experiencias emocionales positivas y negativas se presentaba la Escala de Frecuencia de Experiencias Emocionales Positivas y Negativas, creada ad hoc, que se describe a continuación.

\section{Escala de Frecuencia de Experiencias Emocionales Positivas y Negativas}

Esta escala incluye dos subescalas: la subescala de Frecuencia de experiencias emocionales positivas, compuesta por adjetivos que hacen referencia, en conjunto, a 20 experiencias emocionales positivas, y la subescala de Frecuencia de experiencias emocionales negativas, compuesta por 22 experiencias emocionales negativas. Ejemplos de ítems positivos son "satisfecha" y "alegre", y ejemplos de ítems negativos, "triste" y "ansiosa". A los participantes se les pide que indiquen en una escala de 6 puntos con qué frecuencia $(1=$ nunca, $6=$ siempre) se sienten de la forma que indica cada ítem.

La interpretación de las puntuaciones de cada una de las subescalas, así como de cada una de las variables que las componen, es a mayor puntuación en cada una de ellas, mayor frecuencia de experiencias emocionales positivas o negativas.

La Escala de Frecuencia de Experiencias Emocionales Positivas y Negativas fue validada en un estudio previo (Pascual, Apodaca, Mayordomo y Etxebarria, en revisión). En dicho estudio encontramos datos adecuados de consistencia interna y validez. La consistencia interna de las escalas fue de .94 en el caso de la variable frecuencia de experiencias emocionales positivas y de .93 en el caso de la variable frecuencia de experiencias emocionales negativas. En cuanto a su validez, se comprobó una buena validez de constructo al compararla con el Cuestionario de Salud General (Goldberg e Hillier, 1979). Las puntuaciones de este cuestionario aportan una estimación del grado de severidad de la ausencia de salud mental. Es decir, a mayor puntuación en este cuestionario, peor salud mental. Para hallar la validez de constructo, se realizaron correlaciones de Pearson entre las dos subescalas que componen esta escala (Frecuencia de experiencias emocionales positivas y Frecuencia de experiencias emocionales negativas) y el GHQ. Los resultados mostraron una correlación de $.75(p=.01)$ entre la subescala Frecuencia de experiencias emocionales negativas y el cuestionario GHQ y una correlación de -.80 ( $p=.01)$ entre la subescala de Frecuencia de experiencias emocionales positivas y el GHQ. Por otra parte, los resultados del análisis factorial confirmaron la estructura bidimensional de la escala, siendo el total del porcentaje de varianza explicada de $45.96 \%$ (de $21.57 \%$ en el caso de la variable frecuencia de experiencias emocionales positivas y de $24.39 \%$ en el caso de la variable frecuencia de experiencias emocionales negativas). 


\section{Procedimiento}

A las participantes les llevó media hora aproximadamente rellenar el cuestionario que se acaba de describir.

El protocolo del presente estudio fue aprobado por la Comisión de Ética en Investigación y Docencia (CEID) así como por cada uno de los centros en los que fueron administradas las pruebas. En los casos en los que la participante era menor de edad, se solicitó el consentimiento informado de la familia. En todos los casos se les recordaba que la información solicitada era anónima y confidencial.

\section{Procedimiento estadístico}

Primeramente, para ver si había diferencias, por una parte, entre las mujeres con TCA y las que no tenían estos trastornos y, por otra, entre las mujeres con distintos tipos de TCA en las variables sociodemográficas edad y ocupación, se realizaron pruebas t-test y de chi-cuadrado en el primer caso, y análisis de varianza y la prueba de chi-cuadrado en el segundo.

Por otro lado, para ver si había diferencias entre las mujeres con TCA y las del grupo control en la frecuencia con la que tenían experiencias emocionales positivas y negativas en general, se realizaron pruebas $t$-test comparando el grupo de TCA y el de control en las variables Frecuencia de experiencias emocionales positivas y Frecuencia de experiencias emocionales negativas. Asimismo, se realizaron pruebas t-test con cada una de las variables de frecuencia de experiencias emocionales positivas y frecuencia de experiencias emocionales negativas.

Para ver si había diferencias en la frecuencia con la que las mujeres con distintos tipos de TCA tenían experiencias emocionales positivas y negativas, se realizaron análisis de varianza y pruebas post hoc de Scheffé comparando los distintos grupos de TCA (AN inespecífica, BN purgativa, BN no purgativa y $\mathrm{BN}$ inespecífica) en las variables Frecuencia de experiencias emocionales positivas y Frecuencia de experiencias emocionales negativas. Por último, para realizar un análisis más afinado, se realizaron análisis de varianza y pruebas post hoc de Scheffé comparando los distintos grupos de TCA en cada una de las variables de frecuencia de experiencias emocionales positivas y frecuencia de experiencias emocionales negativas.

\section{Resultados}

Comparaciones entre las mujeres con TCA y las del grupo control en las variables sociodemográficas edad y ocupación

No se encontraron diferencias estadísticamente significativas entre el grupo de TCA y el de control ni en la variable edad, $t(1,196)=1.16, p=.247$, ni en la variable ocupación, $\chi^{2}(6, N=196)=7.4, p=.285$ (véase Tabla 1$)$.

\section{Comparaciones en función del diagnóstico alimentario} en las variables sociodemográficas edad y ocupación

En este caso, tampoco se encontraron diferencias estadísticamente significativas entre los distintos grupos de TCA (AN inespecífica, BN purgativa, BN no purgativa $\mathrm{y}$ $\mathrm{BN}$ inespecífica) ni en la variable edad, $F(3,97)=.43, p=$ .731 , ni en la variable ocupación, $\chi^{2}(15, N=97)=23.11, p$ $=.082$ (véase Tabla 2$)$.

Tabla 1. Datos sociodemográficos de la muestra total.

\begin{tabular}{llll}
\hline & TA & Control & Total \\
\cline { 2 - 4 } Ocupación & $n(\%)$ & $n(\%)$ & $n(\%)$ \\
\hline Estudiante & $69(71.1)$ & $82(82.8)$ & $151(77)$ \\
$\begin{array}{l}\text { Empresaria o directiva } \\
\text { Profesional }\end{array}$ & $1(1)$ & $0(0)$ & $1(0.5)$ \\
$\begin{array}{l}\text { Mis labores } \\
\text { Técnico o mando inter- } 0\end{array}$ & $11(11.3)$ & $5(5.1)$ & $16(8.2)$ \\
medio & $3(3.1)$ & $1(1)$ & $4(2)$ \\
$\begin{array}{l}\text { Empleada administrativa } \\
\text { o de servicios }\end{array}$ & $10(10.3)$ & $9(9.1)$ & $1(0.5)$ \\
$\begin{array}{l}\text { Trabajadora industrial } \\
\text { operaria }\end{array}$ & $\mathrm{u} 3(3.1)$ & $1(1)$ & $4(2)$ \\
\hline & $M(D T)$ & $M(D T)$ & $M(D T)$ \\
Edad & $22.2(5.87)$ & $21.34(4.54)$ & $21.77(5.24)$ \\
\hline
\end{tabular}

Tabla 2. Datos sociodemográficos de los distintos grupos de TCA

\begin{tabular}{|c|c|c|c|c|c|}
\hline & AN inespecífica & BN purgativa & BN no purgativa & $\mathrm{BN}$ inespecífica & Total \\
\hline Ocupación & $n(\%)$ & $n(\%)$ & $n(\%)$ & $n(\%)$ & $n(\%)$ \\
\hline Estudiante & $15(68.2)$ & $11(64.7)$ & $14(82.4)$ & $29(70.7)$ & $69(71.1)$ \\
\hline Empresaria o directiva & 0 & 0 & 0 & $1(2.4)$ & $1(1)$ \\
\hline Profesional & $6(27.3)$ & 0 & $2(11.8)$ & $3(7.3)$ & $11(11.3)$ \\
\hline Mis labores & $1(4.5)$ & $1(5.9)$ & 0 & $1(2.4)$ & $3(3.1)$ \\
\hline Técnico o mando intermedio & 0 & 0 & 0 & 0 & 0 \\
\hline Empleada administrativa o de servicios & 0 & $5(29.4)$ & $1(5.9)$ & $4(9.8)$ & $10(10.3)$ \\
\hline \multirow[t]{3}{*}{ Trabajadora industrial u operaria } & 0 & 0 & 0 & $3(7.3)$ & $3(3,1)$ \\
\hline & $M(D T)$ & $M(D T)$ & $M(D T)$ & $M(D T)$ & $M(D T)$ \\
\hline & $22.64(6.67)$ & $22.76(6.13)$ & $20.76(5.12)$ & $22.33(5.73)$ & $22.2(5.87)$ \\
\hline
\end{tabular}


Tabla 3. Diferencias entre los grupos de TCA y de Control en Frecuencia de experiencias emocionales positivas y Frecuencia de experiencias emocionales negativas (puntuaciones factoriales).

\begin{tabular}{|c|c|c|c|c|c|c|c|c|c|c|}
\hline & \multicolumn{3}{|c|}{ TCA } & \multicolumn{3}{|c|}{ Control } & \multicolumn{2}{|c|}{ Pruebas $t$-test } & \multicolumn{2}{|c|}{ Tamaño del efecto } \\
\hline & $M$ & $D T$ & $n$ & $M$ & DT & $n$ & $g l$ & $t$ & $p$ & Eta $^{2}$ \\
\hline F. exp. emoc. positivas & -.99 & 1.07 & 98 & .2 & .83 & 100 & 196 & -8.7 & .0001 & .28 \\
\hline F. exp. emoc. negativas & 1.32 & 1.25 & 98 & -.41 & .64 & 100 & 196 & 12.32 & .0001 & .44 \\
\hline
\end{tabular}

F. exp. emoc. positivas $=$ Frecuencia de experiencias emocionales positivas; F. exp. emoc. negativas $=$ Frecuencia de experiencias emocionales negativas.

Comparaciones entre las mujeres con TCA y las del Distintas variables de Frecuencia de experiencias emocionales posigrupo control en las variables objeto de estudio

Variables Frecuencia de experiencias emocionales positivas y Frecuencia de experiencias emocionales negativas

Como se puede observar en la Tabla 3, las mujeres con TCA obtuvieron puntuaciones significativamente más bajas que las mujeres sin TCA en la variable Frecuencia de experiencias emocionales positivas y puntuaciones significativamente más altas que éstas en la variable Frecuencia de experiencias emocionales negativas. El tamaño del efecto se calculó mediante el coeficiente Eta ${ }^{2}$. En la Tabla 3 se presentan los coeficientes obtenidos. tivas

En cuanto a las distintas variables de Frecuencia de experiencias emocionales positivas, como se puede observar en la Tabla 4, se encontraron diferencias estadísticamente significativas entre el grupo de TCA y el de control prácticamente en todas las variables; concretamente, en todas menos en las relativas a sentirse Independiente y sentirse Extrovertida. Las mujeres con TCA puntuaron más bajo que las mujeres del grupo control en todas las experiencias emocionales de carácter positivo, aunque en las dos variables citadas las diferencias no alcanzaron significatividad estadística. En la Tabla 4 se presentan los coeficientes Eta $a^{2}$ obtenidos.

Tabla 4. Diferencias entre los grupos de TCA y de Control en la frecuencia de cada una de las experiencias emocionales positivas.

\begin{tabular}{|c|c|c|c|c|c|c|c|c|c|c|}
\hline & \multicolumn{3}{|c|}{ TCA } & \multicolumn{3}{|c|}{ Control } & \multicolumn{3}{|c|}{ Pruebas $t$-test } & \multirow{2}{*}{$\frac{\text { Tamaño del efecto }}{E t a^{2}}$} \\
\hline & $M$ & $D T$ & $n$ & $M$ & $D T$ & $n$ & $g l$ & $t$ & $p$ & \\
\hline Alegre & 3.49 & 1.2 & 97 & 4.43 & .93 & 99 & 194 & -6.14 & .0001 & .16 \\
\hline Capaz & 3.23 & 1.16 & 97 & 4.09 & 1.02 & 100 & 195 & -5.56 & .0001 & .14 \\
\hline Aceptada & 3.56 & 1.31 & 97 & 4.53 & .94 & 99 & 194 & -5.97 & .0001 & .16 \\
\hline Comprendida & 2.96 & 1.11 & 97 & 3.95 & .9 & 99 & 194 & -6.89 & .0001 & .2 \\
\hline Con alta autoestima & 2.18 & 1.24 & 97 & 3.41 & 1.12 & 100 & 195 & -7.33 & .0001 & .22 \\
\hline Estable & 2.89 & 1.22 & 96 & 4.06 & .99 & 99 & 193 & -7.4 & .0001 & .22 \\
\hline Feliz & 3.33 & 1.35 & 98 & 4.35 & 1.04 & 99 & 195 & -5.99 & .0001 & .16 \\
\hline Positiva & 2.85 & 1.32 & 97 & 3.89 & 1.14 & 99 & 194 & -5.93 & .0001 & .15 \\
\hline Satisfecha & 2.84 & 1.13 & 96 & 3.91 & .89 & 97 & 191 & -7.28 & .0001 & .22 \\
\hline Segura & 2.63 & 1.22 & 98 & 3.71 & 1.08 & 100 & 196 & -6.59 & .0001 & .18 \\
\hline Tranquila & 2.67 & 1.16 & 98 & 3.85 & 1.13 & 100 & 196 & -7.24 & .0001 & .21 \\
\hline Con sensación de control & 2.86 & 1.06 & 98 & 3.79 & 1.04 & 99 & 195 & -6.2 & .0001 & .17 \\
\hline Afortunada & 3.44 & 1.43 & 98 & 4.43 & 1.08 & 99 & 195 & -5.52 & .0001 & .14 \\
\hline Activa & 3.84 & 1.29 & 98 & 4.34 & 1.08 & 100 & 196 & -2.97 & .003 & .04 \\
\hline Esperanzada & 3.2 & 1.18 & 98 & 3.84 & 1.2 & 100 & 196 & -3.76 & .0001 & .07 \\
\hline Entusiasmada & 3.17 & 1.18 & 98 & 3.92 & 1.01 & 99 & 195 & -4.76 & .0001 & .1 \\
\hline Con fuerza de voluntad & 3.15 & 1.38 & 97 & 4.14 & 1.21 & 100 & 195 & -5.34 & .0001 & .13 \\
\hline Querida & 3.87 & 1.23 & 98 & 4.61 & .97 & 100 & 196 & -4.71 & .0001 & .1 \\
\hline Independiente & 3.67 & 1.48 & 98 & 4.03 & 1.2 & 100 & 196 & -1.87 & .064 & .02 \\
\hline Extrovertida & 3.67 & 1.47 & 97 & 3.75 & 1.2 & 100 & 195 & -.42 & .676 & .01 \\
\hline
\end{tabular}

Distintas variables de Frecuencia de experiencias emocionales negativas

En cuanto a las distintas variables de Frecuencia de experiencias emocionales negativas, como se puede observar en la Tabla 5, se encontraron diferencias estadísticamente significativas entre el grupo de TCA y el de control en todas las variables, excepto en la relativa a sentirse Exigente con los demás, en la que la diferencia fue tendencial. Como se esperaba, fueron las mujeres con TCA las que puntuaron significativamente más alto que las mujeres del grupo control en todas las variables. En la Tabla 5 se presentan los coeficientes Eta ${ }^{2}$ obtenidos. 
Tabla 5. Diferencias entre los grupos de TCA y de Control en la frecuencia de cada una de las experiencias emocionales negativas.

\begin{tabular}{|c|c|c|c|c|c|c|c|c|c|c|}
\hline & \multicolumn{3}{|c|}{ TCA } & \multicolumn{3}{|c|}{ Control } & \multicolumn{3}{|c|}{ Pruebas $t$-test } & \multirow{2}{*}{$\frac{\text { Tamaño del efecto }}{E t a^{2}}$} \\
\hline & $\bar{M}$ & $D T$ & $n$ & $M$ & $D T$ & $n$ & $g l$ & $t$ & $p$ & \\
\hline Angustiada & 3.58 & 1.23 & 98 & 2.36 & .75 & 99 & 195 & 8.38 & .0001 & .27 \\
\hline Ansiosa & 3.92 & 1.23 & 97 & 2.37 & .85 & 97 & 192 & 10.2 & .0001 & .35 \\
\hline Con sent. inferioridad & 3.87 & 1.5 & 98 & 2.22 & .96 & 99 & 195 & 9.18 & .0001 & .3 \\
\hline Con sent. odio ti misma & 3.49 & 1.49 & 98 & 1.62 & .9 & 99 & 195 & 10.71 & .0001 & .37 \\
\hline Culpable & 3.79 & 1.37 & 98 & 2.31 & .63 & 98 & 194 & 9.7 & .0001 & .33 \\
\hline Desesperada & 3.29 & 1.34 & 96 & 2.12 & .86 & 99 & 193 & 7.29 & .0001 & .22 \\
\hline Enfadada contigo misma & 3.78 & 1.18 & 98 & 2.35 & .74 & 100 & 196 & 10.19 & .0001 & .35 \\
\hline Estresada & 3.96 & 1.27 & 98 & 3.01 & 1.08 & 100 & 196 & 5.68 & .0001 & .14 \\
\hline Fracasada & 3.15 & 1.48 & 97 & 1.74 & .75 & 99 & 194 & 8.47 & .0001 & .27 \\
\hline Hipersensible & 4.38 & 1.38 & 96 & 3.16 & 1.29 & 99 & 193 & 6.35 & .0001 & .17 \\
\hline Irritable & 3.68 & 1.14 & 98 & 2.56 & .87 & 99 & 195 & 7.82 & .0001 & .24 \\
\hline Obsesiva & 3.94 & 1.36 & 98 & 2 & .85 & 98 & 194 & 11.96 & .0001 & .43 \\
\hline Triste & 3.64 & 1.13 & 98 & 2.62 & .69 & 100 & 196 & 7.68 & .0001 & .23 \\
\hline Decepcionada contigo & 3.86 & 1.32 & 98 & 2.22 & .96 & 100 & 196 & 9.98 & .0001 & .34 \\
\hline Con sent. odio otros & 2.43 & 1.15 & 98 & 1.75 & .72 & 99 & 195 & 4.99 & .0001 & .11 \\
\hline Decepcionada con otros & 3.17 & 1.14 & 98 & 2.61 & .92 & 99 & 195 & 3.84 & .0001 & .07 \\
\hline Vacía & 3.16 & 1.49 & 97 & 1.98 & .89 & 99 & 194 & 6.77 & .0001 & .19 \\
\hline Sola & 3.2 & 1.42 & 97 & 2.19 & .9 & 100 & 195 & 5.97 & .0001 & .15 \\
\hline Avergonzada & 3.41 & 1.27 & 97 & 2.66 & 1 & 100 & 195 & 4.63 & .0001 & .1 \\
\hline Exigente contigo misma & 4.56 & 1.24 & 98 & 4.05 & 1.24 & 100 & 196 & 2.89 & .004 & .04 \\
\hline Exigente con los demás & 3.46 & 1.22 & 98 & 3.15 & 1.13 & 100 & 196 & 1.85 & .066 & .02 \\
\hline Impulsiva & 3.69 & 1.38 & 97 & 3.21 & 1.05 & 98 & 193 & 2.72 & .007 & .04 \\
\hline
\end{tabular}

Con sent. inferioridad = Con sentimientos de inferioridad; Con sent. odio ti misma $=$ Con sentimientos de odio hacia ti misma; Decepcionada contigo $=$ Decepcionada contigo misma; Con sent. odio otros = Con sentimientos de odio hacia otros.

Comparaciones en función del diagnóstico alimentario en las variables objeto de estudio

Variables Frecuencia de experiencias emocionales positivas y Frecuencia de experiencias emocionales negativas

Como se puede observar en la Tabla 6 , los resultados mostraron un efecto global significativo tanto en la variable Frecuencia de experiencias emocionales positivas como en la de Frecuencia de experiencias emocionales negativas. En dicha tabla se pueden observar también los tamaños del efecto obtenidos.

Respecto a las diferencias entre los distintos grupos de TCA, en la variable Frecuencia de experiencias emocionales positivas se encontraron diferencias estadísticamente significativas entre el grupo de $\mathrm{AN}$ inespecífica y el de $\mathrm{BN}$ inespecífica $(p=.004)$ y entre el grupo de AN inespecífica y el de $\mathrm{BN}$ no purgativa $(p=.016)$. En ambos casos el grupo de AN inespecífica obtuvo puntuaciones significativamente más bajas. En el caso de la variable Frecuencia de experiencias emocionales negativas también se encontraron diferencias estadísticamente significativas entre el grupo de AN inespecífica y el de $\mathrm{BN}$ inespecífica $(p=.003)$ y entre el grupo de $\mathrm{AN}$ inespecífica y el de BN no purgativa $(p=.028)$. En ambos casos, el grupo de $\mathrm{AN}$ inespecífica obtuvo puntuaciones significativamente más altas.

Tabla 6. Diferencias entre los distintos grupos de TCA en Frecuencia de experiencias emocionales positivas y Frecuencia de experiencias emocionales negativas (puntuaciones factoriales).

\begin{tabular}{|c|c|c|c|c|c|c|c|c|c|c|c|c|c|c|c|c|}
\hline & \multicolumn{3}{|c|}{ AN inespecífica } & \multicolumn{3}{|c|}{ BN purgativa } & \multicolumn{3}{|c|}{ BN no purgativa } & \multicolumn{3}{|c|}{ BN inespecífica } & \multicolumn{3}{|c|}{ Anovas } & \multirow{2}{*}{$\begin{array}{l}\text { Tamaño } \\
\text { del efecto } \\
\text { Eta }\end{array}$} \\
\hline & $\bar{M}$ & $D T$ & $n$ & $M$ & DT & $n$ & $M$ & $D T$ & $n$ & $M$ & $D T$ & $n$ & $N$ & $F$ & $p$ & \\
\hline F. exp. emoc. positivas & -1.69 & .76 & 22 & -1.2 & .99 & 17 & -.62 & 1.22 & 17 & -.68 & 1.02 & 42 & 98 & 5.87 & .001 & .16 \\
\hline F. exp. emoc. negativas & 2.1 & 1.21 & 22 & 1.67 & 1.21 & 17 & .94 & 1.27 & 17 & .93 & 1.08 & 42 & 98 & 5.97 & .001 & .16 \\
\hline
\end{tabular}

F. exp. emoc. positivas $=$ Frecuencia de experiencias emocionales positivas; F. exp. emoc. negativas $=$ Frecuencia de experiencias emocionales negativas.

Distintas variables de Frecuencia de experiencias emocionales positivas

Como se puede observar en la Tabla 7 , se encontró un efecto global significativo en la mayoría de las variables de frecuencia de experiencias emocionales positivas (concretamente, en 13 de ellas). En general, de forma consistente con lo que ocurría en las variables anteriores, las mujeres que tenían con mayor frecuencia diversas experiencias emocionales positivas eran las que tenían $\mathrm{BN}$ inespecífica y las que tenían $\mathrm{BN}$ no purgativa. Por el contrario, las mujeres que tenían con menor frecuencia diversas vivencias emocionales positivas eran las que tenían AN inespecífica. En la Tabla 7 se presentan los coeficientes Eta ${ }^{2}$ obtenidos. 
Tabla 7. Diferencias entre los distintos grupos de TCA en la frecuencia de cada una de las experiencias emocionales positivas.

\begin{tabular}{|c|c|c|c|c|c|c|c|c|c|c|c|c|c|c|c|c|}
\hline & \multicolumn{3}{|c|}{ AN inespecífica } & \multicolumn{3}{|c|}{$\mathrm{BN}$ purgativa } & \multicolumn{3}{|c|}{ BN no purgativa } & \multicolumn{3}{|c|}{$\mathrm{BN}$ inespecífica } & \multicolumn{3}{|c|}{ Anovas } & \multirow{2}{*}{$\begin{array}{r}\text { Tamaño } \\
\text { del efecto } \\
\text { Eta }^{2}\end{array}$} \\
\hline & $M$ & DT & $n$ & $M$ & $D T$ & $n$ & $M$ & $D T$ & $n$ & $M$ & $D T$ & $n$ & $N$ & $F$ & $p$ & \\
\hline Alegre & 2.64 & .9 & 22 & 3.53 & 1.23 & 17 & 3.88 & 1.22 & 17 & 3.78 & 1.13 & 41 & 97 & 5.91 & .001 & .16 \\
\hline Capaz & 2.86 & 1.11 & 21 & 3.12 & .93 & 17 & 3.41 & 1.18 & 17 & 3.38 & 1.25 & 42 & 97 & 1.16 & .329 & .04 \\
\hline Aceptada & 3.05 & 1.02 & 21 & 3.82 & .95 & 17 & 3.82 & 1.55 & 17 & 3.6 & 1.42 & 42 & 97 & 1.58 & .201 & .05 \\
\hline Comprendida & 2.27 & .77 & 22 & 2.76 & .75 & 17 & 3.12 & .93 & 17 & 3.34 & 1.28 & 41 & 97 & 5.38 & .002 & .15 \\
\hline Con alta autoestima & 1.41 & .59 & 22 & 1.76 & .83 & 17 & 2.76 & 1.56 & 17 & 2.51 & 1.27 & 41 & 97 & 6.71 & .0001 & .18 \\
\hline Feliz & 2.5 & .96 & 22 & 3.06 & 1.43 & 17 & 3.82 & 1.42 & 17 & 3.67 & 1.26 & 42 & 98 & 5.28 & .002 & .14 \\
\hline Positiva & 2.14 & .77 & 22 & 2.94 & 1.25 & 17 & 3.24 & 1.48 & 17 & 3.02 & 1.41 & 41 & 97 & 3.09 & .031 & .09 \\
\hline Satisfecha & 2.27 & .94 & 22 & 2.82 & 1.07 & 17 & 3 & 1.32 & 16 & 3.1 & 1.09 & 41 & 96 & 2.84 & .042 & .09 \\
\hline Segura & 1.91 & .68 & 22 & 2.29 & .92 & 17 & 3.35 & 1.58 & 17 & 2.86 & 1.18 & 42 & 98 & 6.35 & .001 & .17 \\
\hline Tranquila & 2.18 & .8 & 22 & 2.18 & 1.07 & 17 & 2.88 & 1.27 & 17 & 3.05 & 1.17 & 42 & 98 & 4.46 & .006 & .13 \\
\hline Con sensación de control & 2.95 & 1.09 & 22 & 2.41 & .94 & 17 & 3.12 & 1.17 & 17 & 2.88 & 1.04 & 42 & 98 & 1.42 & .243 & .04 \\
\hline Afortunada & 2.82 & 1.26 & 22 & 3.12 & 1.45 & 17 & 3.82 & 1.24 & 17 & 3.74 & 1.48 & 42 & 98 & 2.85 & .042 & .08 \\
\hline Esperanzada & 2.5 & .67 & 22 & 3.18 & 1.38 & 17 & 3.47 & 1.01 & 17 & 3.48 & 1.25 & 42 & 98 & 3.96 & .011 & .11 \\
\hline Entusiasmada & 2.45 & .86 & 22 & 3.12 & 1.45 & 17 & 3.29 & 1.1 & 17 & 3.52 & 1.11 & 42 & 98 & 4.42 & .006 & .12 \\
\hline Con fuerza de voluntad & 4 & 1.41 & 21 & 2.59 & 1.5 & 17 & 3.12 & 1.17 & 17 & 2.98 & 1.24 & 42 & 97 & 4.21 & .008 & .12 \\
\hline Querida & 3.36 & 1.18 & 22 & 3.94 & 1.14 & 17 & 4.29 & 1.26 & 17 & 3.93 & 1.24 & 42 & 98 & 2.02 & .116 & .06 \\
\hline Independiente & 3.27 & 1.52 & 22 & 3.59 & 1.33 & 17 & 3.47 & 1.59 & 17 & 4 & 1.45 & 42 & 98 & 1.37 & .258 & .04 \\
\hline Extrovertida & 2.68 & 1.13 & 22 & 3.71 & 1.57 & 17 & 4.12 & 1.54 & 17 & 4 & 1.36 & 41 & 97 & 5.11 & .003 & .14 \\
\hline
\end{tabular}

Respecto a las diferencias entre los distintos grupos de TCA, se encontraron diferencias estadísticamente significativas entre el grupo de $\mathrm{AN}$ inespecífica y el de $\mathrm{BN}$ inespecífica en las variables Alegre $(p=.003)$, Comprendida $(p=.003)$, Con alta autoestima $(p=.006)$, Feliz $(p=.009)$, Segura $(p=$ $.022)$, Tranquila $(p=.035)$, Entusiasmada $(p=.007)$, Con fuerza de voluntad $(p=.043)$ y Extrovertida $(p=.007)$. En todos los casos, el grupo de $\mathrm{BN}$ inespecífica obtuvo puntuaciones más altas que el de AN inespecífica (excepto en la variable Con fuerza de voluntad, en la que ocurría lo contrario). Asimismo, se encontraron diferencias estadísticamente significativas entre el grupo de $\mathrm{AN}$ inespecífica y el de $\mathrm{BN}$ no purgativa en las variables Alegre $(p=.010)$, Con alta autoestima $(p=.005)$, Feliz $(p=.018)$, Segura $(p=.002)$ y Extrovertida $(p=.020)$. En todos los casos, el grupo de BN no purgativa obtuvo puntuaciones más altas que el de AN inespecífica. Por último, se encontraron diferencias estadísticamente significativas entre el grupo de AN inespecífica y el de $\mathrm{BN}$ purgativa en la variable Con fuerza de voluntad $(p=$ .016). Concretamente, el grupo de AN inespecífica obtuvo una puntuación más alta que el de $\mathrm{BN}$ purgativa en esta variable.

\section{Distintas variables de Frecuencia de experiencias emocionales nega- tivas}

Como se puede observar en la Tabla 8, también se encontró un efecto global significativo en muchas de las variables de frecuencia de experiencias emocionales negativas (concretamente, en 10). De forma congruente con los resultados que se acaban de citar, en general, las mujeres con $\mathrm{AN}$ inespecífica eran las que tenían experiencias emocionales ne- gativas con mayor frecuencia, y las mujeres con BN inespecífica y $\mathrm{BN}$ no purgativa, las que tenían experiencias emocionales negativas con menor frecuencia. En la Tabla 8 se presentan los coeficientes Eta ${ }^{2}$ obtenidos.

Respecto a las diferencias entre los distintos grupos de TCA, se encontraron diferencias estadísticamente significativas entre el grupo de AN inespecífica y el de BN inespecífica en las variables Con sentimientos de inferioridad $(p=.020)$, Con sentimientos de odio hacia ti misma $(p=.001)$, Culpable $(p=.016)$, Enfadada contigo misma $(p=.014)$, Obsesiva $(p=.033)$, Triste $(p=.001)$, Decepcionada contigo misma $(p$ $=.030)$, Exigente contigo misma $(p=.027)$ e Impulsiva $(p=$ .012). En todos los casos, el grupo de AN inespecífica obtuvo puntuaciones más altas que el de $\mathrm{BN}$ inespecífica (excepto en la variable Impulsiva, en la que ocurría lo contrario). También se encontraron diferencias estadísticamente significativas entre el grupo de AN inespecífica y el de BN no purgativa en las variables Con sentimientos de inferioridad $(p=$ $.028)$, Triste $(p=.001)$, Decepcionada contigo misma $(p=$ $.046)$, Vacía $(p=.017)$ e Impulsiva $(p=.004)$. En todos los casos, el grupo de AN inespecífica obtuvo puntuaciones más altas que el de BN no purgativa, excepto de nuevo en la variable Impulsiva, en la que el grupo de $\mathrm{BN}$ (no purgativa, en este caso) puntuaba más alto. Asimismo, se encontraron diferencias estadísticamente significativas entre el grupo de $\mathrm{AN}$ inespecífica y el de BN purgativa en la variable Impulsiva $(\phi=.009)$, puntuando nuevamente más alto el grupo de BN (purgativa, en este caso). Por último, también se encontraron diferencias estadísticamente significativas entre el grupo de $\mathrm{BN}$ purgativa y el de $\mathrm{BN}$ inespecífica en la variable Con sentimientos de odio hacia ti misma $(p=.016)$, puntuando el grupo de $\mathrm{BN}$ purgativa más alto. 
Tabla 8. Diferencias entre los distintos grupos de TCA en la frecuencia de cada una de las experiencias emocionales negativas.

\begin{tabular}{|c|c|c|c|c|c|c|c|c|c|c|c|c|c|c|c|c|}
\hline & \multicolumn{3}{|c|}{ AN inespecífica } & \multicolumn{3}{|c|}{ BN purgativa } & \multicolumn{3}{|c|}{ BN no purgativa } & \multicolumn{3}{|c|}{ BN inespecífica } & \multicolumn{3}{|c|}{ Anovas } & \multirow{2}{*}{$\begin{array}{c}\text { Tamaño } \\
\text { del efecto } \\
E t a^{2}\end{array}$} \\
\hline & $\bar{M}$ & $D T$ & $n$ & $M$ & $\overline{D T}$ & $n$ & $M$ & DT & $n$ & $M$ & $\overline{D T}$ & $n$ & $N$ & $F$ & $p$ & \\
\hline Angustiada & 4.05 & 1.25 & 22 & 3.94 & 1.2 & 17 & 3.24 & 1.15 & 17 & 3.33 & 1.2 & 42 & 98 & 2.66 & .053 & .08 \\
\hline Ansiosa & 3.95 & 1.29 & 22 & 4.41 & 1.06 & 17 & 3.88 & 1.22 & 17 & 3.71 & 1.25 & 41 & 97 & 1.34 & .267 & .04 \\
\hline Con sentimientos de inferioridad & 4.77 & 1.23 & 22 & 3.94 & 1.43 & 17 & 3.35 & 1.62 & 17 & 3.57 & 1.43 & 42 & 98 & 4.32 & .007 & .12 \\
\hline Con sentimientos de odio hacia ti misma & 4.36 & 1.53 & 22 & 4.12 & 1.05 & 17 & 3.35 & 1.5 & 17 & 2.83 & 1.31 & 42 & 98 & 7.61 & .0001 & .2 \\
\hline Culpable & 4.64 & 1.14 & 22 & 3.65 & 1.62 & 17 & 3.53 & 1.33 & 17 & 3.5 & 1.25 & 42 & 98 & 4.03 & .010 & .11 \\
\hline Desesperada & 3.77 & 1.63 & 22 & 3.59 & 1.42 & 17 & 3 & 1.27 & 17 & 3.03 & 1.07 & 40 & 96 & 2.10 & .106 & .06 \\
\hline Enfadada contigo misma & 4.41 & 1.01 & 22 & 4.18 & 1.07 & 17 & 3.41 & 1.23 & 17 & 3.43 & 1.13 & 42 & 98 & 5.09 & .003 & .14 \\
\hline Estresada & 4.41 & 1.1 & 22 & 4 & 1.32 & 17 & 4.12 & 1.27 & 17 & 3.64 & 1.28 & 42 & 98 & 1.95 & .128 & .06 \\
\hline Fracasada & 3.64 & 1.65 & 22 & 3.69 & 1.4 & 16 & 2.82 & 1.19 & 17 & 2.83 & 1.45 & 42 & 97 & 2.52 & .063 & .08 \\
\hline Hipersensible & 4.43 & 1.25 & 22 & 4.82 & 1.51 & 17 & 4.59 & 1.28 & 17 & 4.07 & 1.4 & 41 & 96 & 1.42 & .242 & .04 \\
\hline Irritable & 4 & 1.27 & 22 & 4.12 & .93 & 17 & 3.59 & 1.28 & 17 & 3.38 & 1.01 & 42 & 98 & 2.55 & .061 & .08 \\
\hline Obsesiva & 4.64 & 1.26 & 22 & 4.18 & 1.55 & 17 & 3.65 & 1.46 & 17 & 3.6 & 1.17 & 42 & 98 & 3.5 & .018 & .1 \\
\hline Triste & 4.45 & 1.06 & 22 & 3.94 & .9 & 17 & 3.06 & 1.03 & 17 & 3.33 & 1.05 & 42 & 98 & 8.2 & .0001 & .21 \\
\hline Decepcionada contigo misma & 4.59 & 1.05 & 22 & 4.06 & 1.3 & 17 & 3.41 & 1.42 & 17 & 3.57 & 1.29 & 42 & 98 & 4.02 & .010 & .11 \\
\hline Con sentimientos de odio hacia otros & 2.5 & 1.22 & 22 & 2.29 & 1.36 & 17 & 2.94 & 1.43 & 17 & 2.24 & .82 & 42 & 98 & 1.65 & .183 & .05 \\
\hline Decepcionada con otros & 3.32 & .95 & 22 & 3.12 & 1.45 & 17 & 3.12 & 1.27 & 17 & 3.14 & 1.07 & 42 & 98 & .15 & .929 & .01 \\
\hline Vacía & 3.86 & 1.7 & 22 & 3.35 & 1.58 & 17 & 2.35 & 1.22 & 17 & 3.05 & 1.28 & 41 & 97 & 3.76 & .013 & .11 \\
\hline Sola & 3.86 & 1.42 & 22 & 3.29 & 1.16 & 17 & 2.76 & 1.3 & 17 & 2.98 & 1.47 & 41 & 97 & 2.63 & .055 & .08 \\
\hline Avergonzada & 4.05 & 1.17 & 22 & 3.35 & 1.41 & 17 & 3.12 & 1.22 & 17 & 3.22 & 1.21 & 41 & 97 & 2.56 & .059 & .08 \\
\hline Exigente contigo misma & 5.09 & 1.31 & 22 & 4.94 & .83 & 17 & 4.59 & 1 & 17 & 4.12 & 1.31 & 42 & 98 & 3.96 & .010 & .11 \\
\hline Exigente con los demás & 3.55 & 1.22 & 22 & 3.76 & 1.6 & 17 & 3.47 & 1.01 & 17 & 3.29 & 1.13 & 42 & 98 & .67 & .573 & .02 \\
\hline Impulsiva & 2.68 & 1.25 & 22 & 4.12 & 1.36 & 17 & 4.24 & 1.2 & 17 & 3.83 & 1.28 & 41 & 97 & 6.41 & .001 & .17 \\
\hline
\end{tabular}

\section{Discusión}

El objetivo de este estudio era conocer la experiencia emocional de las mujeres con distintos tipos de TCA. En este sentido, habíamos planteado cuatro hipótesis y, como hemos podido observar, todas ellas han sido confirmadas por nuestros resultados.

Si tuviéramos que resumir, de alguna manera, los resultados más importantes de este estudio, diríamos que, en comparación con las mujeres que no tienen TCA, las que sí los tienen experimentan con mayor frecuencia emociones negativas y con menor frecuencia emociones positivas. Asimismo, entre las mujeres con distintos tipos de TCA, son las mujeres con $\mathrm{AN}$ inespecífica y las que tienen $\mathrm{BN}$ purgativa las que peor se encuentran a nivel emocional, y las que tienen $\mathrm{BN}$ inespecífica y $\mathrm{BN}$ no purgativa, las que mejor se encuentran a nivel emocional. El diseño del presente estudio no nos permite saber si este perfil emocional que presentan las mujeres con TCA es "causa" o "consecuencia" del propio trastorno. Sin embargo, pensamos que los resultados que hemos obtenido sí nos permiten plantear algunas hipótesis interesantes a este respecto. En el trabajo de Pascual y sus colaboradores (2011b), mencionado anteriormente, se encontraron resultados muy similares a éstos. Concretamente, los resultados mostraron que las mujeres con TCA, en comparación con las mujeres sin estos trastornos, mostraban mayor dificultad para identificar y describir los sentimientos (alexitimia), una actitud más negativa hacia la expresión emocional, una percepción más negativa de las emociones y formas más desadaptativas de regular las emociones (mayor utilización de la evitación, el escape, la negación, etc.). Asimismo, al comparar a las mujeres con distintos tipos de TCA, se encontró que las mujeres con $\mathrm{AN}$ inespecífica y con $\mathrm{BN}$ purgativa, en comparación con las mujeres con $\mathrm{BN}$ no purgativa y con $\mathrm{BN}$ inespecífica, mostraban igualmente índices más altos de alexitimia, una actitud más negativa hacia la expresión emocional, una percepción más negativa de las emociones y formas más desadaptativas de regular las emociones. Todos estos resultados nos llevan a pensar que las mujeres que padecen estos trastornos tienen dificultades con el manejo y regulación de las emociones. En realidad, esta idea es totalmente consistente con el planteamiento señalado en la introducción- de que los TCA constituyen una forma de regular las emociones (Alpers y TuschenCaffier, 2001; Arnow et al., 1992; Corstorphine, 2006; Heatherton y Baumeister, 1991; Moreno, 2003; Rodríguez et al., 2007; Stice et al, 1996; Waters et al, 2001). Los resultados relativos a las diferencias emocionales entre las mujeres con distintos tipos de TCA son igualmente consistentes con el planteamiento que se acaba de mencionar. En este sentido, cabría pensar, por ejemplo, que cuanto mayor es la frecuencia de emociones negativas, mayores esfuerzos o esfuerzos más severos tiene que hacer la persona para manejar la situación. Así, las personas que peor se encuentran a nivel emocional son las que llevan a cabo una restricción alimentaria severa (AN inespecífica) y las que después de realizar atracones, utilizan métodos de purga, como el vómito, los laxantes y los diuréticos (BN purgativa). Y, por otra parte, las personas que mejor se encuentran a nivel emocional son las que, después de realizar atracones, utilizan conductas compensatorias más aceptadas socialmente (como la dieta o la 
práctica de ejercicio físico) (BN no purgativa), y las que no realizan atracones ni tienen conductas compensatorias con tal regularidad (BN inespecífica). No obstante, y, volviendo a insistir en la idea antes señalada, este estudio no nos permite saber qué es "causa" y qué "consecuencia": si el problema emocional o el TCA. No podemos saber, por ejemplo, si la persona que realiza una restricción alimentaria severa ha desarrollado este problema porque tenía dificultades con sus emociones o si, como consecuencia del propio TCA, la persona tiene dificultades emocionales. Pensamos que seguramente ambas posiciones sean válidas de alguna manera, pero esto, habría que estudiarlo con un diseño más apropiado.

Otro de los resultados que consideramos destacable es el de las diferencias significativas halladas entre los grupos de $\mathrm{AN}$ inespecífica y $\mathrm{BN}$ inespecífica en muchas de las variables contempladas. Al inicio del estudio ya sospechábamos que habría diferencias emocionales claras entre los casos inespecíficos de AN y de BN. Por ello, decidimos analizar estos dos grupos de forma separada. Como hemos podido observar, los resultados obtenidos en el presente estudio avalan plenamente esta decisión: precisamente son éstos los grupos que muestran las diferencias más claras en la mayoría de las variables consideradas. Si los hubiésemos agrupado, tal y como se hace en el DSM-IV-TR (American Psychiatric Association, 2002), se hubiera desdibujado totalmente el perfil emocional de cada uno de estos trastornos. Este resultado, así como el de Pascual y sus colaboradores (2011b), señalado anteriormente, confirma la idea defendida por varios autores (entre ellos, Williamson et al., 2002) respecto la mezcla tan confusa que supone la categoría TCANE del DMS-IV-TR (American Psychiatric Association, 2002). Pensamos que es necesario seguir estudiando esta cuestión en mayor profundidad, para ver, si, efectivamente, esta categoría debería sufrir alguna modificación.

Además de ésta, el presente estudio tiene otras implicaciones clínicas importantes. A la luz de los resultados obtenidos, pensamos que sería conveniente que en los programas de tratamiento de estos trastornos se utilizaran técnicas dirigidas a trabajar las emociones. Nos parece fundamental que las personas que padecen estos trastornos tomen conciencia

\section{Referencias}

Alpers, G.W. y Tuschen-Caffier, B. (2001). Negative feelings and the desire to eat in bulimia nervosa. Eating Behaviors, 2, 339-352.

American Psychiatric Association (2002). Manual diagnóstico y estadístico de los trastornos mentales ( $4^{\mathrm{a}} \mathrm{ed}$., texto revisado). Barcelona: Masson.

Arnow, B., Kenardy, J. y Agras, W. S. (1992). Binge eating among the obese: A descriptive study. Journal of Behavioral Medicine, 15, 155-170.

Blinder, B. J., Cumella, E. J. y Sanathara, V. A. (2006). Psychiatric comorbidities of female inpatients with eating disorders. Psychosomatic Medicine, 68, 454-462.

Breaux, C. A. y Moreno, J. K. (1994). Comparing anorectics and bulimics on measures of depression, anxiety, and anger. Eating Disorders: The Journal of Treatment and Prevention, 2, 158-167.

Bruch, H. (1973). Eating disorders: Obesity, anorexia nervosa and the person within. Nueva York: Basic Books.

Burney, J. e Irwin, H. J. (2000). Shame and guilt in women with eatingdisorder symptomatology. Journal of Clinical Psychology, 56, 51-61. de sus propias emociones, que aprendan a identificarlas, a describirlas, pero, sobre todo, a manejarlas y regularlas de forma adecuada. De esta forma, y siguiendo con el planteamiento antes señalado, al tener un mayor control de sus emociones, pensamos que quizás no necesitarían recurrir al control del peso y de la alimentación para manejar el malestar emocional.

A continuación señalaré algunas de las limitaciones más importantes de este estudio. Por una parte, respecto a la muestra, concretamente respecto a la asignación de la muestra clínica a los distintos grupos de TCA, pensamos que hubiese sido conveniente no realizarla mediante autoinformes, sino a través de una entrevista clínica. Por otro lado, a la hora de interpretar los resultados relativos a las diferencias en la experiencia emocional entre los diferentes grupos, hubiera sido deseable haber controlado posibles variables confundidoras, mediadoras o moderadoras. En este sentido, nos parece que hubiera sido importante, por ejemplo, controlar variables como el nivel de depresión, de ansiedad, etc., ya que es conocido el alto grado de comorbilidad existente entre los TCA y los trastornos del estado de ánimo y de ansiedad (Blinder, Cumella y Sanathara, 2006). Asimismo, nos parece que hubiera sido adecuado evaluar otro tipo de variables como, por ejemplo, el cociente intelectual de las participantes.

Dejando a un lado las limitaciones que tiene el estudio, pensamos que éste nos muestra una fotografía bastante detallada de las emociones que sienten las mujeres con TCA. No obstante, vemos necesario seguir estudiando este tema en mayor profundidad, utilizando un diseño que nos permita concretar de forma más segura el papel que desempeñan las emociones en estos trastornos. Nosotros hemos planteado algunas hipótesis en este sentido, pero, es necesario ponerlas a prueba en futuros trabajos. Como hemos señalado anteriormente, estudiar este tema resulta de sumo interés de cara al tratamiento de estos trastornos, pero también de cara a la prevención de los mismos, ya que los problemas emocionales que parecen tener estas personas puede que también hayan contribuido al desarrollo del trastorno.

Claes, L., Vandereycken, W. y Vertommen, H. (2002). Impulsive and compulsive traits in eating disordered patients compared with controls. Personality and Individual Differences, 32, 707-714.

Corstorphine, E. (2006). Cognitive-emotional-behavioural therapy for the eating disorders: Working with beliefs about emotions. European Eating Disorders Review, 14, 448-461.

Fahy, T. y Eisler, I. (1993). Impulsivity and eating disorders. British Journal of Psychiatry, 162, 193-197.

Forbush, K. y Watson, D. (2006). Emotional inhibition and personality traits: A comparison of women with anorexia, bulimia, and normal controls. Annals of Clinical Psychiatry, 18, 115-121.

Goldberg, D. P. e Hillier, V. F. (1979). A scaled version of the General Health Questionnaire. Psychological Medicine, 9, 139-145.

Heatherton, T. F. y Baumeister, R. (1991). Binge eating as escape from selfawareness. Psychological Bulletin, 110, 86-108.

Izard, C. E. (1972). Patterns of emotions. Nueva York: Academic Press. 
Moreno, S. (2003). Ansia por la comida y trastornos de la conducta alimentaria. Granada: Universidad de Granada.

Nagata, T., Kawarada, Y., Kiriike, N. e Iketani, T. (2000). Multi-impulsivity of Japanese patients with eating disorders: Primary and secondary impulsivity. Psychiatry Research, 94, 239-250.

Norman, D. K. y Herzog, D. B. (1983). Bulimia, anorexia nervosa, and anorexia nervosa with bulimia: A comparative analysis of MMPI profiles. International Journal of Eating Disorders, 2, 43-52.

Overton, A., Selway, S., Strongman, K. y Houston, M. (2005). Eating disorders-The regulation of positive as negative emotion experience. Journal of Clinical Psychology in Medical Settings, 12, 39-56.

Pascual, A., Apodaca, P., Mayordomo, S. y Etxebarria, I. (en revisión). Estudio de validez de la Escala de Frecuencia de Experiencias emocionales positivas y negativas.

Pascual, A., Etxebarria, I., Cruz, M. S. y Echeburúa, E. (2011). Las variables emocionales como factores de riesgo de los trastornos de la conducta alimentaria: un estudio empírico. International Journal of Clinical and Health Psychology, 11, 229-247.

Pascual, A., Etxebarria, I. y Cruz, M. S. (2011). Emotional differences between women with different types of eating disorders. International Journal of Psychology and Psychological Therapy, 11, 1-11.

Pells, J. J. (2005). The role of negative affect and emotional avoidance in the maintenance of binge eating disorder. Maine: Universidad de Maine.

Rodríguez, S., Mata, J. L. y Moreno, S. (2007). Psicofisiología del ansia por la comida y la bulimia nerviosa. Clínica y Salud, 18, 99-118.

Schmidt, U. y Treasure, J. (2006). Anorexia nervosa: Valued and visible. A cognitive-interpersonal maintenance model and its implications for research and practice. British Journal of Clinical Psychology, 45, 343-366.
Shapiro, D. H., Blinder, B. J., Hagman, J. y Pituck, S. (1993). A psychological "sense of control" profile of patients with anorexia nervosa and bulimia nervosa. Psychological Reports, 73, 531-541.

Stice, E., Nemeroff, C. y Shaw, H. E. (1996). Test of the dual pathway model of bulimia nervosa: Evidence for dietary restraint and affect regulation mechanisms. Journal of Social and Clinical Psychology, 15, 340363.

Tiller, J., Schmidt, U., Ali, S. y Treasure, J. (1995). Patterns of punitiveness in women with eating disorders. International Journal of Eating Disorders, 17, 365-371.

Viñuales, M., Fernández, F., Jiménez, S., Turón, V. y Vallejo, J. (2001). Baja autoestima, restricción alimentaria y variables psicopatológicas en anorexia y bulimia nerviosa. Un estudio de casos y controles. Psicología Conductual, 9, 267-278.

Waters, A., Hill, A. y Waller, G. (2001). Bulimics' response to food cravings: Is binge-eating a product of hunger or emotional state? Behaviour Research and Therapy, 39, 877-886.

Way, K. (1993). Anorexia nervosa and recovery: A bunger for meaning. Binghamton, Nueva York: Haworth Press.

Williamson, D. A., Womble, L. G., Smeets, M. A. M., Netemeyer, R. G., Thaw, J. M., Kutlesic, V. et al. (2002). Latent structure of eating disorder symptoms: A factor analytic and taxometric investigation. American Journal of Psychiatry, 159, 412-418.

(Articulo recibido: 06-06-2010, revisado: 22-12-2011, aceptado: 31-12-2011) 